\title{
HIDE-AND-SEARCH GAME WITH THE RISK CRITERION
}

\author{
Kōji Iida . Ryūsuke Hohzaki Kenjyō Satō \\ National Defense Academy
}

(Received May 18, 1993; Revised July 5, 1994)

\begin{abstract}
In this paper, a hide-and-search game with the risk criterion is investigated. The target space consists of $n$ discrete regions and each region is characterized by the searching cost, the detection rate per unit searching effort and the reward when the target is detected. At the beginning of the game, a target selects a region so as to maximize the expected risk of the search and hides himself in it. On the other hand, a searcher searches the target allocating his available total searching cost among regions so as to minimize the expected risk of the search. We formulate this search situation as a two-person zero-sum game and derive optimal strategies.
\end{abstract}

\section{Introduction}

Since Koopman [8] pioneered the search theory, studies on the optimal search have been investigated by many authors. On analysis of optimal search, the previous authors adopted various criteria of the search such as the detection probability of the target, the expected time or cost to detection, the expected risk or reward, the whereabouts probability and so on. As for the optimal search with the criterion of the detection probability or the expected time in one-sided search, many authors have investigated the problems thoroughly and the results are compiled by Stone [11]. Furthermore, the hide-and-search game (one stage search game) with these criteria were also studied by many authors [1]. However, we cannot find so many papers with other criteria. In this paper, we study the optimal search in a two-sided search with the expected risk criterion. Here, the definition of the expected risk is that the expected search cost until detection or stopping of the search, whichever comes first, minus the expected reward gained by the detection of the target. The expected risk criterion is useful to analyze not only the optimal allocation of searching effort but also the optimal starting and the optimal stopping of the search. As for the optimal search under this criterion, only problems of one-sided search are studied $[2,3,6,7,10]$. However, we cannot find any study on the optimal two-sided search with the expected risk criterion.

In this paper, we formulate a hide-and-search game with the expected risk and derive the optimal strategies of the searcher and the target. In the next section, we define the system parameters of the search and assumptions of the model are described in detail. In Section 3, we formulate the problem as a two-person zero-sum game and optimal strategies are derived in Section 4. In Section 5, special cases of our game are examined. Finally in Section 6, several comments on the results presented in this paper are discussed.

\section{Definitions of the System Parameters and Assumptions of the Model}

Assumptions of our model and system parameters used in this paper are described as follows. 
(1) The target space consists of $n$ discrete regions : $\{i ; i=1,2, \cdots, n\}$. The regions are called boxes.

(2) The target selects a box at the beginning of the game and hides himself in it.

The target is assumed to be stationary during the search.

(3) Total search cost $C(>0)$ is available to the searcher and he searches the target allocating $C$ among boxes. Here, the total searching cost $C$ is assumed to be continuously divisible in allocating it among the boxes and the search in box $i \operatorname{costs} \mathcal{C}_{i}(>0)$ per unit searching effort.

(4) The searching effort allocated to box $i$ is denoted by $\phi_{i}(\geqq 0)$. We assume that the effort is allocated in each box simultaneously and the search cost is imposed at the beginning of the search.

(5) If the target exists in box $i$, the conditional probability of detecting the target with unit searching effort is assumed to be $\alpha_{i}(>0)$ irrespective of the history of the past search. This assumption implies that the random search is conducted in each box and the conditional detection probability of search $\phi_{i}$ in box $i$ is given by an exponential function; $1-\exp \left(-\alpha_{i} \phi_{i}\right)$.

(6) If the searcher detects the target in box $i$, he gains a reward $R_{i}(>0)$.

(7) The parameters of the search, $c_{i}, R_{i}, \alpha_{i}$ and $C$ are assumed to be known to both players in advance of the game.

(8) The payoff of the game is defined by the risk of the search. Here we assume that both the target and the searcher are opposing against each other completely in a sense that the target desires to maximize the payoff and the searcher wants to minimize it. The omniscient rationality of both players is assumed.

\section{Formulation of the Hide-and-Search Game}

At the first step of formulation of the search game, we define the pure strategies of both players. The target's pure strategy is defined by his selection of box $i$ in which he hides himself at the beginning of the game. On the other hand, the searcher' $s$ pure strategy is defined by the allocation of the searching effort: $\Phi=\left\{\phi_{i}, i=1,2\right.$, $\cdots, n\}$, where constraints : $\Sigma_{i} c_{i} \phi_{i} \leqq C$ and $\phi_{i} \geqq 0$ for any $i$, are imposed on $\Phi$.

The payoff $g(i, \Phi)$ of the target when the strategies $i$ and $\Phi$ are employed by both players, is presented by

$$
\begin{aligned}
g(i, \Phi) & =\left(1-\exp \left(-\alpha_{i} \phi_{i}\right)\right)\left(\sum_{j} c_{j} \phi_{j}-R_{i}\right)+\exp \left(-\alpha_{i} \phi_{i}\right) \Sigma_{j} c_{j} \phi_{j} \\
& =\sum_{j} \mathcal{C}_{j} \phi_{j}-R_{i}\left(1-\exp \left(-\alpha_{i} \phi_{i}\right)\right) .
\end{aligned}
$$

The searcher should not begin his search if $g(i, \Phi)>0$, since the search is expensive compared with its reward in average, and then, obviously $g(i, \Phi=\{0\})=0$. Here, we examine the properties of the payoff $g(i, \Phi)$ and have the next lemmas.

Lemma 1. Let $\Phi^{0}=\left\{\phi_{i}{ }^{0}\right\}$ be the conditionally optimal strategy of the searcher given the target's strategy $i$.

(1) If $R_{i} \alpha_{i} / c_{i} \leqq 1$, the search should not be started: $\Phi^{0}=\{0\}$ and $g\left(i, \Phi^{0}\right)=0$.

(2) Let $C_{i}{ }^{0}=c_{i}\left(\log \left(R_{i} \alpha_{i} / c_{i}\right)\right) / \alpha_{i}$. If $R_{i} \alpha_{i} / c_{i}>1$ and the total searching cost $C$ is larger than $C_{i}{ }^{0}: C>C_{i}{ }^{0}$, the optimal allocation of searching effort to box $i$ is given by

$$
\phi_{i}{ }^{0}=\frac{1}{\alpha_{i}} \log \frac{R_{i} \alpha_{i}}{c_{i}} .
$$

In this case, the search should be stopped at $C_{i}{ }^{0}$ and then

$$
g\left(i, \Phi^{0}\right)=\frac{c_{i}}{\alpha_{i}}\left(1+\log \frac{R_{i} \alpha_{i}}{c_{i}}\right)-R_{i}<0 .
$$


(3) If $R_{i} \alpha_{i} / c_{i}>1$ and $C \leqq C_{i}{ }^{0}$, the search should be stopped by the cost constraint $C$.

$$
\begin{aligned}
& \phi_{i}^{0}=\frac{C}{c_{i}} . \\
& g\left(i, \Phi^{0}\right)=C-R_{i}\left(1-\exp \left(-\frac{\alpha_{i} C}{c_{i}}\right)\right)<0 .
\end{aligned}
$$

Proof: Differentiating $g(i, \Phi)$ with respect to $\phi_{i}$, we obtain

$$
\begin{aligned}
& \frac{\partial g(i, \Phi)}{\partial \phi_{i}}=c_{i}-R_{i} \alpha_{i} \exp \left(-\alpha_{i} \phi_{i}\right), \\
& \frac{\partial g(i, \Phi)}{\partial \phi_{j}}=c_{j}, \quad i \neq j .
\end{aligned}
$$

Since $\partial g(i, \Phi) / \partial \phi_{j}>0, i \neq j$, from the second equation of $(2), g(i, \Phi)$ is minimized by $\phi_{j}=0$ for all $j(\neq i)$, and if $R_{i} \alpha_{i} / c_{i} \leqq 1, \partial g(i, \Phi) / \partial \phi_{i}>0$ for all $\phi_{i}>0$ from the first equation of (2). Hence, $g(i, \Phi)$ is minimized at $\Phi^{0}=\{0\}$, i. e. the search should not be started in this case. On the other hand, if $R_{i} \alpha_{i} / c_{i}>1$, then $\partial g(i, \Phi) / \partial \phi_{i}<0$ at $\phi_{i}=0$ and $\lim _{\phi_{i} \rightarrow \infty} \partial g(i, \Phi) / \partial \phi_{i}=c_{i}>0$. Therefore, if $C$ is sufficiently large, $g(i, \Phi)$ minimized at some positive $\phi_{i}{ }^{0}=\log \left(\left(R_{i} \alpha_{i}\right) / c_{i}\right) / \alpha_{i}$ from $\partial g(i, \Phi) / \partial \phi_{i}=0$ and $\phi_{j}=0$ for all $j(\neq i)$. In this case, the search should be continued until $\Sigma_{j} C_{j} \phi_{j}=C_{i} \phi_{i}=C_{i} \log \left(R_{i} \alpha_{i} / C_{i}\right) / \alpha_{i} \equiv C_{i}{ }^{0}(\langle C)$. However, if the total cost $C$ being available to the searcher is not so much : $C<C_{i}{ }^{0}$, the searcher must stop the search by his cost constraint $C$. (q.e.d.) Lemma 2. $g(i, \Phi)$ is strictly convex in $\Phi$ for any $i$.

Proof. Since $g(i, \Phi)$ is the exponential function of $\phi_{i}$ as shown in Eq. (1), we can easily prove the next inequality for any $\Phi^{1}$ and $\Phi^{2}, \Phi^{1} \neq \Phi^{2}$, satisfying the constraints and for any $\lambda: 0<\lambda<1$.

$$
g\left(i, \lambda \Phi^{1}+(1-\lambda) \Phi^{2}\right)<\lambda g\left(i, \Phi^{1}\right)+(1-\lambda) g\left(i, \Phi^{2}\right) .
$$

As shown in Lemma 2 , our search game is a strictly convex game. The basic theorem of the strictly convex game is quoted from the text [12] without proof.

Lemma 3. In a strictly convex game, the minimizer (the searcher) possesses a unique optimal strategy; moreover, this strategy is pure.

Here, we define the mixed strategy of the target: $P=\left\{p_{i}, i=1,2, \cdots, n\right\}$, where $p_{i}$ is the probability that the target selects box $i$ for his hiding box. The expected payoff $G(P, \Phi)$ of the target is given by

$$
G(P, \Phi)=\Sigma_{i} p_{i} g(i, \Phi)=\Sigma_{i} \mathcal{C}_{i} \phi_{i}-\Sigma_{i} p_{i} R_{i}\left(1-\exp \left(-\alpha_{i} \phi_{i}\right)\right) \text {. }
$$

Therefore, our problem is formulated as the game to obtain the optimal strategy $\left(P^{*}, \Phi^{*}\right)$ satisfying

$$
G\left(P^{*}, \Phi^{*}\right)=\min _{\Phi} \max _{P} G(P, \Phi)=\max _{P} \min _{\Phi} G(P, \Phi) \equiv G .
$$

subject to constraints:

$$
\begin{aligned}
& \sum_{i} c_{i} \phi_{i} \leqq C \text { and } \phi_{i} \geqq 0 \text { for any } i, \\
& \sum_{i} p_{i}=1 \text { and } p_{i} \geqq 0 \text { for any } i .
\end{aligned}
$$

\section{Solution of the Game}

Let $P^{*}=\left\{p_{i}^{*}\right\}$ and $\Phi^{*}=\left\{\phi_{i}^{*}\right\}$ be the optimal strategies of the target and the searcher, respectively. $P^{*}$ and $\Phi^{*}$ are optimal if and only if

$$
G\left(P, \Phi^{*}\right) \leqq G \leqq G\left(P^{*}, \Phi\right) \text {. }
$$

Theorem 1. Let $I^{0}$ be the set of boxes $\left\{i \mid c_{i} /\left(R_{i} \alpha_{i}\right) \geqq 1\right\}$. If there exists some box $k$ belonging to $I^{0}$, the optimal strategy of the target is $P^{*}=\left\{p_{k}{ }^{*}=1, k \in I^{0}\right.$ and $p_{i}{ }^{*}=0$ 
for all $i\}$ and the searcher's optimal strategy is $\Phi^{*}=\left\{\phi_{i}{ }^{*}=0\right.$ for all $\left.i\right\}$. The value of the game is $G=0$. If plural boxes belong to $I^{\circ}$, since the above holds for any $k$ $E I^{0}$, the mixed strategies weighted by arbitrary $p_{k} \geqq 0$ for all $k \in I^{0}$ are also optimal: $P^{*}=\left\{p_{k}{ }^{*} \geq 0\right.$ for any $k \in I^{0}$ and $\left.p_{i}{ }^{*}=0, i \notin I^{0}\right\}$.

Proof: Since $G\left(P, \Phi^{*}=\{0\}\right)=0$ for any $P$, the first inequality of (6) is obvious. The second inequality $G=0 \leqq G\left(P^{*}, \Phi\right)$ is proved by Lemma 1-(1).

(q. e.d.)

Theorem 2. If and only if $c_{i} /\left(R_{i} \alpha_{i}\right)<1$ for all $i$ and $\Sigma_{i}\left\{c_{i} /\left(R_{i} \alpha_{i}\right)\right\} \geqq 1$, the optimal strategy of the target is any $p_{i}{ }^{*}$ such that $P^{*}=\left\{0<p_{i}{ }^{*} \leqq c_{i} /\left(R_{i} \alpha_{i}\right)\right.$ for any $\left.i\right\}$ and the searcher's optimal strategy is $\Phi^{*}=\left\{\phi_{i}^{*}=0\right.$ for all $\left.i\right\}$ : the searcher should not start the search.

Proof: To prove the necessity of the theorem, we assume that $c_{i} /\left(R_{i} \alpha_{i}\right)<1$ for all $i$ and $\sum_{i}\left\{c_{i} /\left(R_{i} \alpha_{i}\right)\right\} \geqq 1$. The first inequality of (6) is proved by substituting $\Phi^{*}=$ $\{0\}$ and showing $G\left(P, \Phi^{*}\right)=0$ for any $P$. To prove the second inequality of (6), we show $\min _{\Phi} G\left(P^{*}, \Phi\right)=G=0$. Differentiating $G\left(P^{*}, \Phi\right)$ with respect to $\phi_{i}$, we obtain $\partial G\left(P^{*}, \Phi\right) / \partial \phi_{i}=c_{i}-p_{i}{ }^{*} R_{i} \alpha_{i} \exp \left(-\alpha_{i} \phi_{i}\right)$. Since $0<p_{i}{ }^{*} \leqq c_{i} /\left(R_{i} \alpha_{i}\right), \partial G\left(P^{*}, \Phi\right) / \partial \phi_{i}$ $>0$ for all $\phi_{i}>0: G\left(P^{*}, \Phi\right)$ is a strictly increasing function of all $\phi_{i}>0$. Therefore, $G\left(P^{*}, \Phi\right)$ is non-negative and has a minimum value at $\Phi=\{0\}$ and $\min _{\Phi} G\left(P^{*}, \Phi\right)=$ $G=0$ is proved.

Next to prove the sufficiency, we assume that the optimal strategies are given by $P^{*}=\left\{0<p_{i}{ }^{*} \leqq c_{i} /\left(R_{i} \alpha_{i}\right)\right\}$ and $\Phi^{*}=\{0\}$. In this case, since any $P^{(i)}$ such that $p^{(i)}=$ $\left\{p_{i}=c_{i} /\left(R_{i} \alpha_{i}\right), 0 \leqq p_{j} \leqq c_{j} /\left(R_{j} \alpha_{j}\right), j \neq i\right\}$ is also optimal for any $i, p_{i}=c_{i} /\left(R_{i} \alpha_{i}\right)<1$ is concluded and $c_{i} /\left(R_{i} \alpha_{i}\right)<1$ for all $\mathrm{i}$ is proved. Then, the inequality $\Sigma_{i}\left\{c_{i} /\left(R_{i} \alpha_{i}\right)\right\} \geqq$ $\sum_{i} p_{i}=1$ is obtained directly.

If $\Sigma_{i}\left\{c_{i} /\left(R_{i} \alpha_{i}\right)\right\}<1$, obviously $c_{i} /\left(R_{i} \alpha_{i}\right)<1$ for all $i$ and the target cannot select such $\left\{p_{i}{ }^{*}\right\}:\left\{0<p_{i}{ }^{*} \leqq C_{i} /\left(R_{i} \alpha_{i}\right)\right\}$, since $\Sigma_{i} p_{i}{ }^{*}<1$. In this case, the next Lemma is proved.

Lemma 4. If $\sum_{i}\left\{c_{i} /\left(R_{i} \alpha_{i}\right)\right\}<1$, the optimal strategies of the target $P^{*}$ and the searcher $\Phi^{*}$ satisfy $p_{i}{ }^{*}>0$ and $\phi_{i}{ }^{*}>0$ for all $i$.

Proof: The contraposition of Theorem 2 is that if $\sum_{i}\left\{c_{i} /\left(R_{i} \alpha_{i}\right)\right\}\left\langle 1\right.$, ( $\left.\exists i, p_{i}{ }^{*}\right\rangle$ $\left.c_{i} /\left(R_{i} \alpha_{i}\right)\right)$ or $\left(\exists i, \phi_{i}{ }^{*}>0\right)$. In the former case, since $\partial G(P, \Phi) /\left.\partial \phi_{i}\right|_{\phi_{i}=0}=c_{i}{ }^{-} p_{i}{ }^{*} R_{i} \alpha_{i}$ $\left\langle 0, \phi_{i}{ }^{*}\right\rangle 0$ is concluded too, and therefore in either case, the searcher should not stop the search immediately if $\Sigma_{i}\left\{c_{i} /\left(R_{i} \alpha_{i}\right)\right\}<1$. The lemma is proved by the reductive absurdity. Here, suppose that there exists an optimal strategy $\Phi^{*}$ of the searcher such as $\phi_{i}{ }^{*}=0$ for some box $i$ and $\phi_{j}{ }^{*}>0$ for any $j(\neq i)$. In this case, since the target should select the box which is not searched, the target's optimal strategy $P^{*}$ opposing the searcher's optimal strategy $\Phi^{*}$ is obviously $\left\{p_{i}{ }^{*}=1, p_{j}{ }^{*}=0, j \neq i\right\}$ and then $G\left(P^{*}, \Phi^{*}\right)$ $=\Sigma{ }_{j} C_{j} \phi_{j}{ }^{*}>0$ holds always from Eq. (3). It contradicts the definition of the searcher's optimal strategy. Hence, $\phi_{i}{ }^{*}>0$ is concluded for all $i$.

Next, suppose $P^{*}$ be a target's optimal strategy with $p_{i}{ }^{*}=0$ for some $i$. Then the searcher's optimal strategy $\Phi^{*}$ opposing $P^{*}$ is $\phi_{i}^{*}=0$ for $i$, since the searcher should not search the box in which the target does not exist certainly. In this case, obviously there exists such box $k: p_{k}{ }^{*}>0$ and $\phi_{k}{ }^{*}>0$, and let $P$ be a target's strategy such as $\left\{p_{i}=\Delta p(>0), p_{k}=p_{k}{ }^{*}-\Delta p, p_{j}=p_{j}{ }^{*}\right.$ for all $j(\neq i, k)$ and $\left.p_{k}{ }^{*}>0\right\}$. Then, we have $G\left(P^{*}, \Phi^{*}\right)$ - $G\left(P, \Phi^{*}\right)=-\Delta p\left(1-\exp \left(-\alpha_{k} \phi_{k}{ }^{*}\right)\right)<0$, and $G\left(P^{*}, \Phi^{*}\right)<G\left(P, \Phi^{*}\right)$ is deduced. It contradicts the definition of the optimal strategy $P^{*}$, hence, $p_{i}{ }^{*}>0$ is concluded. (q. e.d.)

Hereafter, we assume $\sum_{i}\left\{c_{i} /\left(R_{i} \alpha_{i}\right)\right\}<1$. In this case, since $p_{i}{ }^{*}>0$ and $\phi_{i}{ }^{*}>0$ for all $i$ from Lemma 4 , our problem is reduced to obtain $P^{*}$ and $\Phi^{*}$ such that

$$
\begin{aligned}
& \left\{p_{i}^{*}\right\}: \max _{P} G\left(P, \Phi^{*}\right)=G, \\
& \left\{\phi_{i}^{*}\right\}: \min _{\Phi} G\left(P^{*}, \Phi\right)=G,
\end{aligned}
$$


under the constraints (4) and (5). Then, the next lemma is derived.

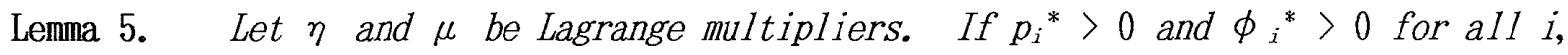
the optimal strategies $\Phi^{*}$ and $P^{*}$ are obtained by equations:

$$
\begin{aligned}
& R_{i}\left(1-\exp \left(-\alpha_{i} \phi_{i}{ }^{*}\right)\right)=\eta \text { for all } i, \\
& c_{i}-p_{i}{ }^{*} \alpha_{i} R_{i} \exp \left(-\alpha_{i} \phi_{i}{ }^{*}\right)=c_{i} \mu \text { for all } i, \\
& \left(C-\sum_{i} c_{i} \phi_{i}{ }^{*}\right) \mu=0,
\end{aligned}
$$

and Eqs. (4) and (5). The value of the game is given by

$$
G=\sum_{i} c_{i} \phi_{i}^{*}-\eta \text {. }
$$

Proof: Since $G\left(P, \Phi^{*}\right)$ is a linear function of $\left\{p_{i}\right\}$ and $p_{i}{ }^{*}>0$ for all $i$ from Lemma 4, Eq. (8) is obtained from the first problem of (7). On the other hand, Eqs. (9) and (10) are obtained from the Kuhn-Tucker theorem since $G\left(P^{*}, \Phi\right)$ is a strictly convex function of $\left\{\phi_{i}\right\}$ for the second problem of (7). Then, we have Eq. (11) from

$$
\max _{P} G\left(P, \Phi^{*}\right)=\min _{\Phi} G\left(P^{*}, \Phi\right)=\Sigma_{i} c_{i} \phi_{i}^{*}-\eta . \quad \text { (q. e. d. ) }
$$

As for the Lagrange multiplier $\mu$ in the above lemma, we can conclude that $\mu \leqq 0$ since $\partial G\left(P^{*}, \Phi^{*}\right) / \partial \phi_{i}{ }^{*} \leqq 0$. If $C>\Sigma_{i} \mathcal{C}_{i} \phi_{i}{ }^{*} \equiv C^{0}, \mu=0$ from Eq. (10) and then we have

$$
c_{i}-p_{i}^{*} \alpha_{i} R_{i} \exp \left(-\alpha_{i} \phi_{i}^{*}\right)=0 \text { for all } i,
$$

from Eq. (9). In this case, the search is stopped at $C^{0}$ before using the total search cost $C$. (The derivation of $C^{0}$ will be shown later.) In either case of stopping the search by $C$ or $C^{0},\left\{\phi_{i}^{*}\right\}$ must satisfy Eq. (8). Therefore, Eq. (9) or (12) is used to determine $p_{i}{ }^{*}$. Let $\eta$ and $\eta_{0}$ be the Lagrange multipliers when the search is stopped by $C$ and $C^{0}$, respectively. By determining the Lagrange multipliers in Lemma 5, we can derive the solution of the game as follows.

Theorem 3. Suppose $\Sigma_{i}\left\{c_{i} /\left(\alpha_{i} R_{i}\right)\right\}<1$. $\eta$ and $\eta_{0}$ are the solutions of the next equations,

$$
\begin{aligned}
& \Sigma_{i} \frac{C_{i}}{\alpha_{i}} \log \left(\frac{R_{i}}{R_{i}-\eta}\right)=C, \\
& \Sigma_{i} \frac{c_{i}}{\alpha_{i}\left(R_{i}-\eta_{0}\right)}=1,
\end{aligned}
$$

respectively. Using $\eta_{0}$, we have the threshold cost $C^{0}$ by

$$
C^{0}=\Sigma_{i} \frac{C_{i}}{\alpha_{i}} \log \left(\frac{R_{i}}{R_{i}-\eta_{0}}\right) .
$$

If $C^{0} \geqq C$, the optimal strategies of the searcher and the target are given by

$$
\begin{aligned}
& \phi_{i}{ }^{*}=\frac{1}{\alpha_{i}} \log \left(\frac{R_{i}}{R_{i}-\eta},\right. \\
& p_{i}^{*}=\frac{c_{i} /\left(\alpha_{i}\left(R_{i}-\eta\right)\right)}{\sum_{i} c_{i} /\left(\alpha_{i}\left(R_{i}-\eta\right)\right)} .
\end{aligned}
$$

The value of the game is obtained by

$$
G=C-\eta
$$

If $C^{0}<C$, the optimal strategies are given by

$$
\begin{aligned}
& \phi_{i}^{*}=\frac{1}{\alpha_{i}} \log \left(\frac{R_{i}}{R_{i}-\eta_{0}}\right), \\
& {p_{i}}^{*}=\frac{c_{i}}{\alpha_{i}\left(R_{i}-\eta_{0}\right)} .
\end{aligned}
$$


The value of the game is given by

$$
G=C^{0}-\eta_{0}
$$

Proof. Let $R_{0}$ be $\min _{i} R_{i}$. For $\eta ; 0 \leqq \eta \leqq R_{0}$, we obtain $\phi_{i}{ }^{*}$ as Eq. (16) from Eq. (8). Hence, if the search is stopped by the constraint of total cost $C\left(C^{0} \geqq C\right)$, Eq. (13) is established. From Eq. (13), we can obtain $\eta$ uniquely. To see it, we set the left-hand side of Eq. (13) as $f(\eta)$. Then, $f(\eta):\left(0, R_{0}\right) \rightarrow(0, \infty)$ and $\mathrm{d} f(\eta) / \mathrm{d} \eta=$ $\Sigma_{i}\left\{c_{i} /\left(\alpha_{i}\left(R_{i}-\eta\right)\right)\right\}>0$ are easily confirmed. Hence, the equation $f(\eta)=C$ has a unique solution $\eta, 0<\eta<R_{0}$. Using $\eta$ obtained from Eq. (13), we can derive $\mu$ and $p_{i}{ }^{*}$ from Eqs. (5), (8) and (9) as follows.

$$
\begin{aligned}
\mu & =1-\frac{1}{\sum_{i}\left\{c_{i} /\left(\alpha_{i}\left(R_{i}-\eta\right)\right)\right\}}, \\
p_{i}{ }^{*} & =\frac{c_{i}(1-\mu)}{\alpha_{i}\left(R_{i}-\eta\right)}=\frac{c_{i} /\left(\alpha_{i}\left(R_{i}-\eta\right)\right)}{\sum_{i}\left\{c_{i} /\left(\alpha_{i}\left(R_{i}-\eta\right)\right)\right\}} .
\end{aligned}
$$

Next, we consider the case that the search is stopped optimally before exhausting the total search cost $C\left(C^{0}\langle C)\right.$, and then, let $\eta_{0}$ be the Lagrange multiplier $\eta$ in Eq. (8). The searcher's optimal strategy; Eq. (16)' is derived from Eq. (8) too. In this case, since $\mu=0$ as described before, Eq. (12) holds from Eq. (10). Then, we obtain Eq. (17)' from Eqs. (8) and (12). Since $C>\Sigma_{i} C_{i} \phi_{i}{ }^{*}$, Eq. (13) does not hold in this case. Hence, we use the constraint (5) to determine $\eta_{0}$ and we have Eq. (14). We can easily show that Eq. (14) has a unique solution as follows. Since $0<\eta_{0}$ $\left\langle R_{0}\right.$ as mentioned before and $\Sigma_{i}\left\{c_{i} /\left(\alpha_{i} R_{i}\right)\right\}<1$ by the assumption of Theorem, $\sum_{i}\left\{c_{i} /\left(\alpha_{i}\left(R_{i}-\eta_{0}\right)\right)\right\}$ is a strictly increasing function of $\eta_{0}$ from $\Sigma_{i}\left\{c_{i} /\left(\alpha_{i} R_{i}\right)\right\}$ $(<1)$ to infinity. Hence, Eq. (17) has a unique solution. Eq. (15) is derived by substituting Eq. (16)' into $C^{0}=\Sigma_{i} C_{i} \phi_{i}{ }^{*}$. The value of the game Eqs. (18) and (18)' are obtained from Eq. (11).

\section{Special Cases of the Game}

In this section, we examine special cases of our game.

If $R_{i}=R$ for all $i$ in our model, the solution of the game is very simplified and the closed form solution is derived easily. Omitting proof, we show the next.

Corollary 3-1. If $R_{i}=R$ for all $i$ and $\Sigma_{i}\left\{c_{i} /\left(\alpha_{i} R\right)\right\}<1$ in Theorem 3 , the solution of the game is as follows.

We define $C^{0}$ as

$$
C^{0}=\log \left(\frac{R}{\Sigma_{i}\left(c_{i} / \alpha_{i}\right)}\right) \Sigma_{i} \frac{c_{i}}{\alpha_{i}}
$$

If $C^{0}<C$, the optimal strategies of the searcher and the target are

$$
\begin{aligned}
& \phi_{i}^{*}=\frac{1}{\alpha_{i}} \log \left(\frac{R}{\Sigma_{i}\left(c_{i} / \alpha_{i}\right)}\right), \\
& {p_{i}}^{*}=\frac{c_{i}}{\alpha_{i} \Sigma_{i}\left(c_{i} / \alpha_{i}\right)} .
\end{aligned}
$$

The value of the game is given by

If $C^{0} \geqq C$, the optimal strategies are

$$
G=C^{0}-R+\Sigma_{i} \frac{C_{i}}{\alpha_{i}}
$$

$$
\begin{aligned}
& \phi_{i}{ }^{*}=\frac{C}{\alpha_{i} \Sigma_{i}\left(c_{i} / \alpha_{i}\right)}, \\
& p_{i}{ }^{*}=\frac{c_{i}}{\alpha_{i} \sum_{i}\left(c_{i} / \alpha_{i}\right)} \text {. }
\end{aligned}
$$


In this case, the value of the game is given by

$$
G=C-R\left\{1-\exp \left(-\frac{C}{\Sigma_{i}\left(C_{i} / \alpha_{i}\right)}\right)\right\} .
$$

Next, we set $c_{i}=1$ and $R_{i}=R \rightarrow \infty$ in Corollary $3-1$. In this case, we have the next Corollary.

Corollary 3-2. If we set $c_{i}=1$ and $R_{i}=R \rightarrow \infty$ for all $i$ in Corollary $3-1$, the optimal strategies $P^{*}$ and $\Phi^{*}$ are obtained as follows.

$$
\begin{aligned}
& \phi_{i}{ }^{*}=\frac{C}{\alpha_{i} \Sigma_{i}\left(1 / \alpha_{i}\right)}, \\
& p_{i}{ }^{*}=\frac{1}{\alpha_{i} \Sigma_{i}\left(1 / \alpha_{i}\right)} .
\end{aligned}
$$

The result of Corollary 3-2 is completely identical with the optimal strategies of the hide-and-search game with the criterion of detection probability of the target studied previously by Gittins and Roberts [5] or Nakai [9]. This correspondence is elucidated as follows. The constraint of the total searching cost $C$ becomes $C \geqq \Sigma_{i} \phi_{i}$ (the total searching effort) by setting $c_{i}=1$, and if we set $R_{i} \equiv R$, the target space becomes homogeneous with respect to the cost-and-reward structure. Furthermore, if we set $R \rightarrow \infty$, the search is not stopped before exhausting the total searching effort $C$. Therefore, the problem of minimizing the expected risk becomes identical with the problem of maximizing the detection probability of the target under the constraint of the searching effort. This problem is just the same as the previous studies $[5,9]$. This is the reason why the solution of special case of our game coincides with the results obtained by the previous authors.

Next, setting $R \rightarrow \infty$ in Corollary $3-1$, we deal with the cumulative searching cost $C$ as a variable increasing sequentially from zero until the detection of the target. Let $\psi_{i}{ }^{*}(C)$ be the optimal density of searching effort in box $i$ at $C$ and $\phi_{i}{ }^{*}=\int_{0}^{C} \psi_{i}{ }^{*}(x) d x$. Then, the next corollary is obtained.

Corollary 3-3. Suppose $R_{i} \equiv R$ and $R$ is sufficiently large. We consider the case in which cumulative searching cost $C$ increases from zero continuously with rate $\triangle C$ and the search is continued until detection of the target satisfying $\sum_{i} c_{i} \phi_{i}{ }^{*}=C$ in each time. Then, the optimal strategies are

$$
\begin{aligned}
& {\psi_{i}}^{*}(C)=\frac{1}{\alpha_{i} \Sigma_{i}\left(c_{i} / \alpha_{i}\right)}, \\
& p_{i}^{*}=\frac{c_{i}}{\alpha_{i} \sum_{i}\left(c_{i} / \alpha_{i}\right)} .
\end{aligned}
$$

Let $p_{i}{ }^{*}(C)$ be the posterior probability of the target being in box $i$ when the target is not found until $C$, the next relation holds.

$$
\frac{\alpha_{i} p_{i}^{*}(\mathcal{C})}{C_{i}}=\text { constant for any } i \text {. }
$$

Proof. By the assumption: $R_{i}=R$, Corollary $3-1$ is established, and in this corollary, if $R \rightarrow \infty$, then we have $C^{0} \rightarrow \infty$. Hence, the searcher's optimal strategy is always stopped by the cost constraint $C$ and Eqs. (20) and (21) hold. As for the searcher's optimal strategy, Eq. (20) holds and we obtain Eq. (22) by differentiating $\phi_{i}{ }^{*}$ given by Eq. (20) with respect to $C$. Eq. (23) is the same as Eq. (21). Eq. (24) is derived as follows. The posterior probability $p_{i}{ }^{*}(C)$ of the target being in box $i$ when it is not found until $C$ is presented by 


$$
p_{i}^{*}(C)=\frac{p_{i}^{*} \exp \left(-\alpha_{i} \int_{0}^{c} \psi_{i}^{*}(x) d x\right)}{\sum_{i} p_{i}^{*} \exp \left(-\alpha_{i} \int_{0}^{C} \psi_{i}{ }^{*}(x) d x\right)} .
$$

In the above, since $\exp \left(-\alpha_{i} \int_{0}^{C} \psi_{i}{ }^{*}(x) d x\right)=\exp \left(-C / \Sigma_{i}\left(c_{i} / \alpha_{i}\right)\right)$ is obtained from Eq. (22), substituting it into the above, we have $p_{i}{ }^{*}(C)=p_{i}{ }^{*}$. Therefore, Eq. (24) is derived from Eq. (23) as follows.

$$
\frac{\alpha_{i} p_{i}^{*}(C)}{c_{i}}=\frac{\alpha_{i} p_{i}^{*}}{c_{i}}=\frac{1}{\sum_{i}\left(c_{i} / \alpha_{i}\right)} \text {. }
$$

It should be noted that Eqs. (22), (23) and (24) are completely identical with the optimal strategies of the search game with the criterion of the expected cost shown by Gittins [4]. In this case, since the reward is assumed to be sufficiently large, the search is continued until detection and the searcher gains the reward $R$ certainly. Then, to minimize the expected risk means to minimize the expected cost until detection. Hence, the solution given by Corollary 3-3 becomes identical with the solution of Gittins' problem [4].

\section{$\S 7 . \quad$ Discussions}

In this section, we examine the meaning of the optimal conditions and discuss generalizations of the model to be investigated in future.

(1) According to Theorems 1 and 2, the searcher should not begin his search in either case: $\left(\min _{i} c_{i} /\left(R_{i} \alpha_{i}\right) \geqq 1\right)$ or $\left(\Sigma_{i}\left\{c_{i} /\left(R_{i} \alpha_{i}\right)\right\} \geqq 1\right.$ and $c_{i} /\left(R_{i} \alpha_{i}\right)<1$ for all $\left.i\right)$. The implication of Theorem 1 is explained as follows. The value $c_{i} /\left(R_{i} \alpha_{i}\right)$ is the conditional expected cost versus reward ratio when unit searching effort is allocated to box $i$ given the target being in the box really. Hence, if this value is not smaller than 1 , the search in box $i$ is too expensive for the searcher. If there exists such box, since the target selects always the box by the assumption of the omniscient rationality of the target, the searcher is always compelled to do the expensive search. Therefore, if $\min _{i} c_{i} /\left(R_{i} \alpha_{i}\right) \geqq 1$, the searcher should not begin the search to avoid the expensive search. On the other hand, if the condition of Theorem $2: c_{i} /\left(R_{i} \alpha_{i}\right)<1$ for all $i$, such box mentioned above does not exist. However, if $\Sigma_{i}\left\{c_{i} /\left(R_{i} \alpha_{i}\right)\right\} \geqq 1$ in this case, the target can select an optimal strategy $\left\{p_{i}{ }^{*}\right\}$ by which the expected risk of the searcher becomes non-negative as stated in the proof of Theorem 2. Hence, since the search is also too expensive in average in this case, the searcher should not begin the search.

0n the other hand, if $c_{i} /\left(R_{i} \alpha_{i}\right)<1$ for all $i$ and $\Sigma_{i}\left\{c_{i} /\left(R_{i} \alpha_{i}\right)\right\}<1$, the optimal strategies of the target and the searcher are $p_{i}{ }^{*}>0$ and $\phi_{i}{ }^{*}>0$ for all $i$ by Lemma $4:$ The searcher should not remain any box unsearched and the target should not eliminate the possibility of hiding in any box. Because, if otherwise in either side, by the assumption of omniscient rationality of the players in the game theory, the searcher or the target can take advantage of the knowledge $p_{i}{ }^{*}=0$ or $\phi_{i}{ }^{*}=0$, respectively, to maximize his own reward.

(2) The interpretations of Eqs. (8), (9) and (10) for the optimal strategy $\phi_{i}{ }^{*}$ in Lemma 5 from the view point of the searcher are stated as follows. Since the left-hand side of Eq. (8) is the conditional expected reward given the target being in box i, Eq. (8) means that the searcher should allocate his searching effort so as to equalize the conditional expected reward to $\eta$ among boxes. By this allocation, the searcher does not give any advantageous box to the target to hide. As for Eq. (9), dividing both sides of Eq. (9) by $c_{i}$, we obtain $\left\{c_{i}-p_{i}{ }^{*} \alpha_{i} R_{i} \exp \left(-\alpha_{i} \phi_{i}{ }^{*}\right)\right\} / c_{i}=\mu$. The left-hand side of this equation is the ratio of the marginal expected risk at $\phi_{i}{ }^{*}$ and the searching 
cost in box $i$. By Theorem for the optimal distribution of searching effort given by Iida [6], the searcher can minimize the overall expected risk by allocating his searching effort so as to equalize the marginal expected risk versus searching cost ratio to $\mu$ among boxes. Therefore, the searcher can gains the minimum risk by allocating his searching effort according to Eq. (9). As for Eq. (10) which is the complementary slackness condition of this non-linear programming problem, this equation elucidates the stopping condition of the search. As stated before, if the search should be stopped by $C^{0}(<0)$, then the marginal expected risk versus cost ratio $\mu$ becomes zero at $C^{0}$, and if the search should be stopped by the cost constraint $C$, the value of $\mu$ is non-positive.

On the other hand, from the viewpoint of the target, the optimal conditions of Lemma 5 are interpreted as follows. Since the conditional risk $g(i, \Phi)$ given the target's box are equalized to $C-\eta$ by Eqs. (1) and (8), the target has not any preferable box to hide. But by determining his distribution by Eq. (9), the target can avoid to give the searcher any efficient box having the low marginal expected risk, and this strategy is effective for the target to maximize the expected risk.

(3) The value $C^{0}$ given by Theorem 3 is the total searching cost on which the marginal expected risk of the search among boxes becomes all zero, namely the optimal stopping point of the search before the cost constraints $C$.

(4) Finally, let us consider problems to be investigated in future. In this paper, we assume a stationary target. If the target can move and if he knows the searcher's current position, the problem is formulated by an evasion-and-search game which is characterized as a multistage game. Theoretical treatment of this problem may be very difficult, but it is an important problem to be studied in future. In this paper, we consider the optimal allocation of the total searching cost $C$. If the search game being constrained by a cost rate in every search time instead of the total cost, the time dependent allocation of searching effort is obtained. As for the search problem of this type with the risk criterion, Iida [6] investigated the one-sided search. The exponential detection function $f(\phi)$ assumed in this paper can be generalized to more general detection functions with regular properties: $\mathrm{d} f(\phi) / \mathrm{d} \phi>0$ and $\mathrm{d}^{2} f(\phi) / \mathrm{d} \phi^{2}$ $<0$ for all $\phi$ without any difficulty. We deal with the discrete target space in this paper. This assumption will be generalized to a continuous target space and the similar optimal solution to Theorem 3 will be derived by the variational calculus approach instead of the Kuhn-Tucker theorem.

\section{Acknowledgments}

The authors are very grateful to referees for their useful comments to refine this paper.

\section{References}

[1] Benkoski, S. J., Monticino, M.G. and Weisinger, J.R.: A Survey of the Search Theory Literature. Naval Research Logistics, 18 (1991), pp. 469-494.

[2] Chew, M. C.Jr. : A Sequential Search Procedure. The Annals of Mathematical Statis tics, 38 (1967), pp. 494-502.

[3] Chew, M. C.Jr. : Optimal Stopping in a discrete Search Problem. Operations Research, 21 (1973), pp. 741-747.

[4] Gittins, J.S. : An Application of Control Theory to a Game of Hide and Seek. International Journal of Control, 30 (1979), pp.981-987. 
[5] Gittins, J.C. and Roberts, D. M. : The Search for an Intelligent Evader Concealed in One of an Arbitrary Number of Regions. Naval Research Logistics Quarterly, 26 (1979), pp. 651-666.

[6] Iida, K.: Optimal Search and Stop in Continuous Search Process. Journal of the Operations Research Society of Japan, 27 (1984), pp. 1-30.

[7] Iida, K. : Studies on the Optimal Search Plan. Springer-Verlag, Berlin, 1992.

[8] Koopman, B. 0. : Search and Screening. OEG Rep. No. 56, 1946.

[9] Nakai, T. : Search Models with Continuous Effort under Various Criteria. Journal of the Operations Research Society of Japan, 31 (1988), pp. 335-352.

[10] Ross, S.M. : A Problem in Optimal Search and Stop. Operations Research, 17 (1969), (1969), pp. 984-992.

[11] Stone, L. D.: Theory of OptimaI Search, Mil.Appl. Sec. of ORSA., 1975.

[12] Vorob' ev, N. N. : Game Theory. Springer-Verlag, 1977.

Ko-ji IIDA: Department of Applied Physics, National Defense Academy, Hashirimizu 1-10-20, Yokosuka, 239, Japan. 